\title{
EDITORIAL
}

\section{Special issue on selected articles from the proceedings of the 2007 Denver X-ray Conference}

The Powder Diffraction journal has published representative papers from the proceedings of the Denver X-ray Conference, Advances in X-ray Analysis, since 2004. This collaboration between Powder Diffraction and the Denver $\mathrm{X}$-ray Conference has increased the number of articles available to the readers of Powder Diffraction and circulation for the authors of Advances in X-ray Analysis. This collaboration has received many positive responses from the X-ray analysis community.

This issue of Powder Diffraction is dedicated to selected high-quality articles from Advances in X-ray AnalysisVolume 51, Proceedings of the 56th Annual Conference on Applications of X-ray Analysis. The conference returned to Colorado Springs 30 July to 3 August 2007 and attracted over 300 registered attendees and more than 200 exhibit personnel.

Conference week began with sixteen tutorial workshops, held on Monday and Tuesday. Topics included X-ray Optics, Diffraction Peak Broadening and Peak Shape Analysis, Fundamentals and Applications of Neutron Scattering for Characterizing Structural Materials and Components, Small Angle Scattering, Rietveld Applications I and II, X-ray Crystallography without Crystals, Two-dimensional XRD, Sample Preparation I and II, Trace Analysis, Analysis of
RoHS/WEEE Elements, Quantitative Analysis I and II, Basic $\mathrm{XRF}$, and Energy Dispersive XRF.

A plenary session, "Stardust-X-rays in Space," on the NASA Stardust mission with four invited presentations as well as sixteen special sessions filled the remaining two and a half days of the conference. Topics included New Developments in XRD and XRF Instrumentation, Stardust Characterization, Detectors and Sources, Microbeam X-ray Analysis, Analysis of Nanomaterials, Diffraction from Biopolymers and Biological Systems, Neutron Analysis, Polymers and Composites, Stress Analysis, Applications of Linear and Area Detectors for XRD, Fusion and Industrial Applications of XRF, Quantitative XRF, Trace Analysis, and Applications of Handheld XRF and Analysis of RoHS/ WEEE Elements.

I thank the editors of Advances in X-ray Analysis, John Anzelmo, Victor Buhrke, John Gilfrich, George Havrilla, Cev Noyan, and Robert Snyder for selecting and editing the articles that appear in this special issue of Powder Diffraction. I also thank Assistant Editor Allen Huang for his extended effort in preparing the papers for publication in Powder Diffraction.
Ting C. Huang Editor-in-Chief 